\title{
Broadband Mid-IR superabsorption with aperiodic polaritonic photonic crystals
}

G. C. R. Devarapu

\author{
S. Foteinopoulou \\ s.foteinopoulou@exeter.ac.uk
}

\author{
School of Physics, College of Engineering, Mathematics and Physical Sciences (CEMPS),University of \\ Exeter, Exeter, EX4 4QL, United Kingdom \\ School of Physics, College of Engineering, Mathematics and Physical Sciences (CEMPS), University of \\ Exeter, Exeter, EX4 4QL, United Kingdom
}

We propose an approach for broadband near-perfect absorption with aperiodic-polaritonic photonic crystals (PCs) operating in the phononpolariton gap of the constituent material. In this frequency regime the bulk polaritonic materials are highly reflective due to the extreme permittivity values, and so their absorption capabilities are limited. However, we are able to achieve absorptance of more than $90 \%$ almost across the entire phonon-polariton gap of SiC with a SiC-air aperiodic one-dimensional(1D)-PC with angular bandwidth that covers the range of realistic diffraction-limited sources. We explore two types of aperiodic PC schemes, one in which the thickness of the SiC layer increases linearly, and one in which the filling ratio increases linearly throughout the structure. We find that the former scheme performs better in terms of exhibiting smoother spectra and employing less SiC material. On the other hand, the second scheme performs better in terms of the required total structure size. We analyze the principles underpinning the broadband absorption merit of our proposed designs, and determine that the key protagonists are the properties of the entry building block and the adiabaticity of the aperiodic sequencing scheme. Further investigation with derivative lamellar sequences,-resulting by interchanging or random positioning of the original building blocks-, underline the crucial importance of the building block arrangement in an increasing order of thickness. If we relax the requirement of near-perfect absorption, we show that an averaged absorption enhancement across the SiC phonon-polariton gap of $\sim 10$ can be achieved with much shorter designs of the order of two free-space wavelengths. Our findings suggest that our aperiodic polaritonic PC route can be promising to design broadband electromagnetic absorbers across the spectrum.

[DOI: http://dx.doi.org/10.2971/jeos.2014.14012]

Keywords: Phonon-polaritons, superabsorbers, photonic crystals, Mid-IR

\section{INTRODUCTION}

Absorbers are crucial components in electromagnetic (EM) wave detection and energy harvesting devices such as photovoltaics [1, 2], thermophotovoltaics [3]-[7], bolometers [8]-[10] and imaging devices [11]-[13]. Therefore, many current research efforts are focusing on optimizing EM absorption with different mechanisms such as near-field enhancement through plasmonic resonances [1, 2, 14, 15] or impedance-matching by metamaterial structures [16]-[19]. In all these schemes the absorptance enhancement in the absorbing material is facilitated by the metallic structures which are integrated with the absorbing material.

An alternative route has been explored with photonic crystals (PCs) relying solely on the excitation of lossy Floquet-Bloch modes in a structure with a single kind of absorbing material [20]-[25]. In the Mid-IR regime mostly PCs with metallic constituents have been explored [23]. However metals have a small skin depth [26], thus thick PC structures would be required to get high absorption enhancement [23].

On the other hand, polaritonic materials have a larger skin depth in the Mid-IR region thus offering the possibility of a larger light-matter interaction path. Therefore polaritonic materials could be a promising constituent materials for engi- neering absorption. However, overcoming the ultrahigh reflection within the phonon-polariton gap, also known as the Reststrahlen band [26] is a major challenge.

However, the recent results of Devarapu and Foteinopoulou [24, 25] demonstrating near-perfect absorption with a $\mathrm{SiC}$ PC system seem promising in this direction. The underlying mechanism was a combination of a quickly vanishing lossy Floquet-Bloch mode $[25,27]$ and tailoring of the energy velocity at the interface $[24,25]$. Careful engineering of such phenomenon has led to a compact-superabsorber design where $90 \%$ of light gets absorbed within the top absorbing layer of thickness $\lambda / 1000$ [25]. Nevertheless the operation bandwidth in the latter PC structure was narrow. An important aspect in many applications such as bolometers and thermophotovoltaics, is to have absorption enhancement over a broad range of wavelengths. Therefore, here we explore a possibility to obtain a broadband near-perfect absorption by utilizing polaritonic PCs. For this purpose we employ aperiodicpolaritonic 1D-PCs with varying building block sizes, inspired by the broadband reflection/antireflection property observed in chirped gratings [28]-[30].

In particular this paper is organized as follows. In Section 2, 


\section{Aperiodic PC scheme 1}

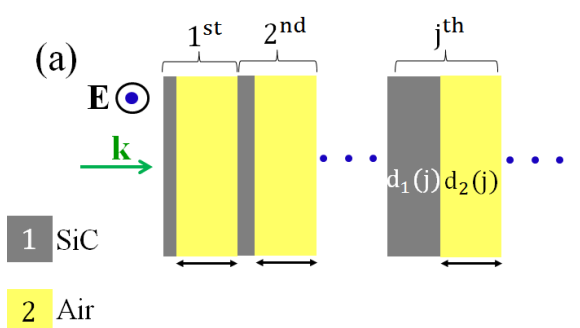

Aperiodic PC scheme 2

(b)

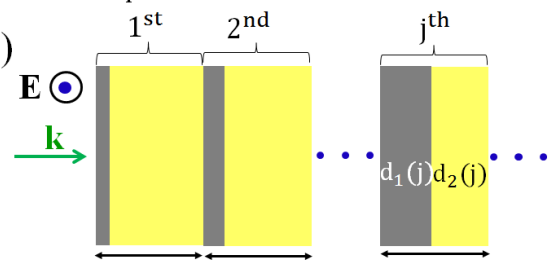

FIG. 1 Schematic diagrams of the two types of aperiodic SiC-air 1D-PC structures. (a) The thickness of the SiC layer in each building block changes linearly while the thickness of the air layers remains the same. (b) The filling ratio in each building block changes linearly while the lattice constant remains the same.

we describe the aperiodic polaritonic 1D-PCs under study. In Section 3, we present the methodology we adopted for evaluating the absorptance performance of the proposed aperiodic PCs. In Section 4, we discuss our results of near-perfect absorptance of the two aperiodic PCs under study and compare their performance. In Section 5, we investigate the robustness of the broadband absorption merit under angular illumination. In Section 6, we determine the key operation principles underpinning the broadband functionality of our proposed designs. In Section 7, we analyse the significance of the particular order of the building blocks in the aperiodic PC. Based on the determined principles, we present in Section 8 , a shrinked aperiodic PC design which demonstrates an averaged, -within the SiC Reststrahlen band-, absorption enhancement with a value close to 10 . Finally, we present our conclusions in Section 9.

\section{SYSTEM UNDER STUDY}

We will consider two aperiodic 1D-PC structures comprising of alternating layers of $\mathrm{SiC}$ and air as shown schematically in Figures 1(a) and 1(b). The respective thicknesses of the $\mathrm{SiC}$ and air layers in the $\mathrm{j}^{\text {th }}$ building block of the aperiodic PC are $d_{1}(j)$ and $d_{2}(j)$. We denote the permittivity of air as $\varepsilon_{2}$, with its value being one. The permittivity of $\mathrm{SiC}$ is denoted as $\varepsilon_{1}$, and modelled with the Lorentzian function [26],

$$
\varepsilon_{1}(\omega)=\varepsilon_{\infty}\left(1+\frac{\omega_{L}^{2}-\omega_{T}^{2}}{\omega_{T}^{2}-\omega^{2}-i \omega \Gamma}\right),
$$

where $\omega$ is the angular frequency of the incident EM wave. The parameters in the Lorentzian function-taken from Ref. [31]-are $\varepsilon_{\infty}=6.7, \omega_{T}=2 \pi \times 23.79 \mathrm{THz}, \omega_{L}=2 \pi \times 29.07 \mathrm{THz}$ and $\Gamma=2 \pi \times 0.1428 \mathrm{THz}$. We have particularly chosen $\mathrm{SiC}$ as the constituent of the aperiodic PCs, since the Reststrahlen band of the $\mathrm{SiC}$ spans from $10.3 \mu \mathrm{m}$ to $12.6 \mu \mathrm{m}$, where many pathogens and bio-molecules have their fingerprints [32]-[34]. Moreover this frequency regime is an important atmospheric
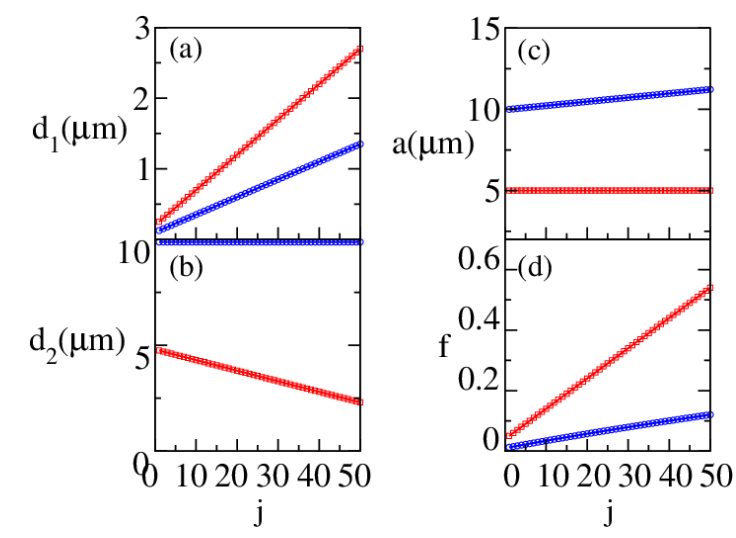

FIG. 2 Structural parameters of the aperiodic PCS of Figure 1(a) (blue circles) and Figure ${ }_{1}$ (b) (red squares) in each building block j. (a) thickness of the SiC layers, (b) thickness of air layers, (c) lattice constant and (d) filling ratio.

N-band window that facilitates the study of astronomical objects that are colder than $500 \mathrm{~K}$ [35]-[37].

In the first type of aperiodic PC shown in Figure 1(a), the thickness of the SiC layer in each building block changes linearly as:

$$
d_{1}(j)=d_{1}(1)+\frac{1}{5}(j-1) \cdot d_{1}(1),
$$

where $\mathrm{d}_{1}(1)=0.125 \mu \mathrm{m}$ is the thickness of the first $\mathrm{SiC}$ layer in the aperiodic PC. The thickness of the air layers $d_{2}$ is $9.875 \mu \mathrm{m}$ and it remains constant throughout the aperiodic PC.

On the other hand, in the second type of aperiodic PC shown in Figure 1(b), the filling ratio for each building block changes linearly. The filling ratio of the $j^{\text {th }}$ building block is given by:

$$
f(j)=f(1)+\frac{1}{100}(j-1),
$$

where $\mathrm{f}(1)=0.05$ is the filling ratio of the first building block. Note, the lattice constant $\mathrm{a}=5 \mu \mathrm{m}$ is constant throughout the aperiodic PC, implying that the thickness of $\mathrm{SiC}$ and air layer in the $j^{\text {th }}$ building block of the PC would be $d_{1}(j)=a \cdot f(j)$ and $\mathrm{d}_{2}(\mathrm{j})=\mathrm{a}-\mathrm{d}_{1}(\mathrm{j})$ respectively.

In Figure 2, we show the variation of the structural parameters from one building block to the next one in the first and second type of the aperiodic PCs as blue circles and red squares respectively. In Figures 2(a) and 2(b), we show the $\mathrm{SiC}$ and air thicknesses respectively in each building block j. In Figures 2(c) and 2(d), we show the lattice constant and filling ratio in each building block $\mathrm{j}$, respectively.

\section{METHODOLOGY}

In this section, we present the methodology we adopted to study the aperiodic SiC-air lamellae sequence. To evaluate the absorption performance of the two types of aperiodic PCs described above, we need spectral information on their absorptance A. For this reason we calculate with the Transfer Matrix Method (TMM) [38]-[40] the absorptance A as A=1-T-R. T and $R$ represent respectively the transmittance and reflectance of 


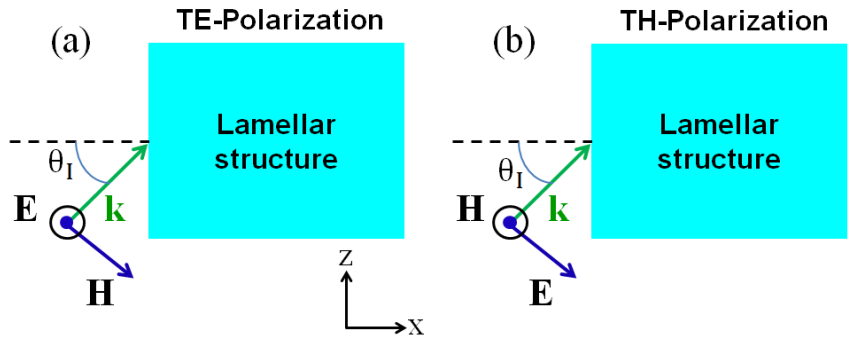

FIG. 3 EM waves incident on a multilayer structure with lamellae along the $y z$-plane (a) TE-polarization case (b) TH-polarization case.

the aperiodic lamellar structures, determined from the transmission and reflection amplitudes as $\mathrm{T}=|t|^{2}$ and $\mathrm{R}=|r|^{2}$ since our entire structure is embedded in air. In particular,

$$
\left[\begin{array}{l}
1 \\
r
\end{array}\right]=M^{\text {total }}\left[\begin{array}{l}
t \\
0
\end{array}\right]
$$

where $M^{\text {total }}$ is the total transfer matrix through the aperiodic $\mathrm{PC}$ with $\mathrm{N}$ building blocks given by:

$$
M^{\text {total }}=\left(\prod_{\mathrm{j}=1}^{\mathrm{N}-1} M^{21} P^{1}(\mathrm{j}) M^{12} P^{2}(\mathrm{j})\right) M^{21} P^{1}(\mathrm{~N}) M^{12} .
$$

In the above equation, $M^{12}$ and $M^{21}$ are the transfer matrices that relate the amplitude of the EM waves going from medium 1 (SiC) to medium 2 (air) and vice versa and are given by:

$$
M^{12}=\frac{1}{2}\left[\begin{array}{cc}
1+\chi^{12} & 1-\chi^{12} \\
1-\chi^{12} & 1+\chi^{12}
\end{array}\right],
$$

and

$$
M^{21}=\frac{1}{2}\left[\begin{array}{cc}
1+\chi^{21} & 1-\chi^{21} \\
1-\chi^{21} & 1+\chi^{21}
\end{array}\right],
$$

where the parameters $\chi^{12}$ and $\chi^{21}$ relate to the SiC permittivity $\varepsilon_{1}$ and angle of incidence $\theta_{I}$ as follows:

(i) For Normal incidence $\left(\theta_{I}=0\right)$ both polarizations are degenerate and we have,

$$
\chi^{12}=\frac{1}{\sqrt{\varepsilon_{1}}} \quad \text { and } \quad \chi^{21}=\sqrt{\varepsilon_{1}} .
$$

(ii) For incidence at an angle $\theta_{I} \neq 0$ and TE polarization [see schematics of Figure 3(a)] we have,

$$
\chi^{12}=\sqrt{\frac{1-\sin ^{2} \theta_{I}}{\varepsilon_{1}-\sin ^{2} \theta_{I}}} \quad \text { and } \quad \chi^{21}=\sqrt{\frac{\varepsilon_{1}-\sin ^{2} \theta_{I}}{1-\sin ^{2} \theta_{I}}} .
$$

(iii) For incidence at an angle $\theta_{I} \neq 0$ and $\mathrm{TH}$ polarization [see schematics of Figure 3(b)] we have,

$$
\chi^{12}=\varepsilon_{1} \sqrt{\frac{1-\sin ^{2} \theta_{I}}{\varepsilon_{1}-\sin ^{2} \theta_{I}}} \text { and } \chi^{21}=\frac{1}{\varepsilon_{1}} \sqrt{\frac{\varepsilon_{1}-\sin ^{2} \theta_{I}}{1-\sin ^{2} \theta_{I}}} .
$$

Then, $P^{1}(\mathrm{j})$ and $P^{2}(\mathrm{j})$ in Eq. (5), are the respective propagation matrices in the $\mathrm{SiC}$ and air layers within the $j^{\text {th }}$ building block and are given by:

$$
P^{1}(\mathrm{j})=\left[\begin{array}{cc}
e^{-i k_{1 x} \mathrm{~d}_{1}(\mathrm{j})} & 0 \\
0 & e^{i k_{1 x} \mathrm{~d}_{1}(\mathrm{j})}
\end{array}\right]
$$

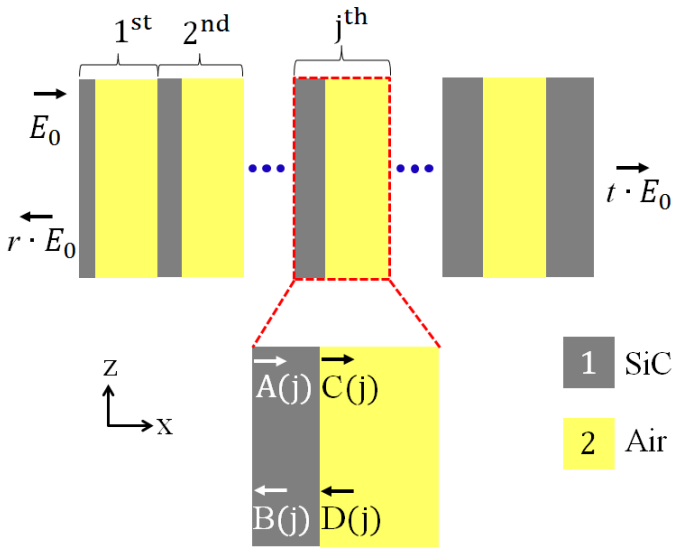

FIG. 4 Schematic of an aperiodic PC indicating field amplitudes upon entrance and exit at normal incidence. The amplitudes within the SiC and air layers of an arbitrary building block $\mathrm{j}$ of the aperiodic $\mathrm{PC}$ are also shown. $E_{0}$ is the incident electric field amplitude.

and

$$
P^{2}(\mathrm{j})=\left[\begin{array}{cc}
e^{-i k_{2 x} \mathrm{~d}_{2}(\mathrm{j})} & 0 \\
0 & e^{i k_{2 x} \mathrm{~d}_{2}(\mathrm{j})}
\end{array}\right]
$$

with $k_{1 x}$ and $k_{2 x}$ representing the $x$-component of the wavevectors inside the $\mathrm{SiC}$ and air layers respectively. Thus, for normal incidence,

$$
k_{1 x}=\sqrt{\varepsilon_{1}} \frac{\omega}{c} \quad \text { and } \quad k_{2 x}=\frac{\omega}{c},
$$

and for off-normal incidence at an angle $\theta_{I} \neq 0$,

$$
k_{1 x}=\sqrt{\varepsilon_{1}-\sin ^{2} \theta_{I}} \frac{\omega}{c} \text { and } k_{2 x}=\sqrt{1-\sin ^{2} \theta_{I}} \frac{\omega}{c},
$$

where $\omega$ represents the frequency of the impinging wave and $c$ represents the vacuum speed of light.

Furthermore, in order to gain insight where the light gets absorbed within the aperiodic PC, we will also look into the electric-field distributions, -for the case of normal incidence. These are given by:

$$
\begin{gathered}
E(x)=e^{i k_{2 x} x}+r e^{-i k_{2 x} x}, \quad \text { for } \quad x \leq 0 \\
E(x)=A(\mathrm{j}) e^{i k_{1 x}\left(x-S_{\mathrm{a}}(\mathrm{j})\right)}+B(\mathrm{j}) e^{-i k_{1 x}\left(x-S_{\mathrm{a}}(\mathrm{j})\right)}, \\
\text { for } S_{\mathrm{a}}(\mathrm{j}) \leq x \leq S_{\mathrm{a}}(\mathrm{j})+\mathrm{d}_{1}(\mathrm{j}) \\
E(x)=C(\mathrm{j}) e^{i k_{2 x}\left(x-S_{\mathrm{a}}(\mathrm{j})-\mathrm{d}_{1}(\mathrm{j})\right)}+D(\mathrm{j}) e^{-i k_{2 x}\left(x-S_{\mathrm{a}}(\mathrm{j})-\mathrm{d}_{1}(\mathrm{j})\right),} \\
\text { for } S_{\mathrm{a}}(\mathrm{j})+\mathrm{d}_{1}(\mathrm{j}) \leq x \leq S_{\mathrm{a}}(\mathrm{j}+1)
\end{gathered}
$$

$E(x)=t e^{i k_{2 x}\left(x-S_{\mathrm{a}}(\mathrm{N}-1)-\mathrm{d}_{1}(\mathrm{~N})\right)}$

$$
\text { for } x \geq S_{\mathrm{a}}(\mathrm{N}-1)+\mathrm{d}_{1}(\mathrm{~N}) \text {. }
$$

With $S_{\mathrm{a}}(\mathrm{j})$, we denote the location of the front $\mathrm{SiC}$ face at the $\mathrm{j}^{\text {th }}$ building block. i.e $S_{\mathrm{a}}(\mathrm{j})=\sum_{\mathrm{i}=1}^{\mathrm{j}-1} \mathrm{a}(\mathrm{i})$ for $\mathrm{j}>1$ and $S_{\mathrm{a}}(\mathrm{j})=0$ for $j=1$, where $a(i)$ is the size of the $i^{\text {th }}$ building block in the aperiodic PC. Moreover, as indicated in Figure $4, A(\mathrm{j})$ and $B(\mathrm{j})$ 
are the electric-field amplitudes of the forward and backward going waves within the SiC layer of the $j^{\text {th }}$ building block in the aperiodic PC. Similarly $C(j)$ and $D(j)$ are the electric-field amplitudes of the forward and backward going waves within the air layer of the $\mathrm{j}^{\text {th }}$ building block. These are determined by the following recursive relations,

$$
\left[\begin{array}{l}
A(\mathrm{j}) \\
B(\mathrm{j})
\end{array}\right]=P^{1}(\mathrm{j}) M^{12} P^{2}(\mathrm{j}) M^{21}\left[\begin{array}{l}
A(\mathrm{j}+1) \\
B(\mathrm{j}+1)
\end{array}\right],
$$

and

$$
\left[\begin{array}{l}
C(\mathrm{j}) \\
D(\mathrm{j})
\end{array}\right]=P^{2}(\mathrm{j}) M^{21} P^{1}(\mathrm{j}+1) M^{12}\left[\begin{array}{l}
C(\mathrm{j}+1) \\
D(\mathrm{j}+1)
\end{array}\right],
$$

with $1 \leq j<N$, together with the use of their values at $j=N$. The latter are given by:

$$
\left[\begin{array}{l}
A(\mathrm{~N}) \\
B(\mathrm{~N})
\end{array}\right]=P^{1}(\mathrm{~N}) M^{12}\left[\begin{array}{l}
t \\
0
\end{array}\right]
$$

and

$$
\left[\begin{array}{l}
C(\mathrm{~N}) \\
D(\mathrm{~N})
\end{array}\right]=\left[\begin{array}{l}
t \\
0
\end{array}\right]
$$

Moreover in Section 6 we analyze how the properties of the aperiodic photonic crystals may correlate with the properties of the corresponding periodic photonic crystals made from the underlying building blocks of the aperiodic lamellae. In particular, we discuss the correlation of the broadband absorption to the coupling to allowed modes in the corresponding periodic PCs.

The photonic modes in a periodic medium are subject to Bloch's theorem just like electrons in a natural crystal [38]-[43]. This means that the fields at the entrance of the $j^{t h}$ cell of a periodic PC structure would be equal to the fields at the entrance of the $(j+1)^{t h}$ cell times a phase factor $e^{-i q a}$ with $\mathrm{q}$ being the Floquet-Bloch wavevector and a the lattice constant of the periodic stack. This means:

$$
\left[\begin{array}{l}
E^{+}(\mathbf{j}) \\
E^{-}(\mathbf{j})
\end{array}\right]=e^{-\mathrm{iqa}}\left[\begin{array}{l}
E^{+}(\mathrm{j}+1) \\
E^{-}(\mathrm{j}+1)
\end{array}\right]
$$

where the superscripts $(+)$ and $(-)$ denote the respective wave amplitudes along the $+x$ - and $-x$ - direction.

In purely dielectric media the Bloch wave vector $\mathrm{q}$ is purely real in regimes of allowed EM propagation and completely imaginary outside [38, 42, 43]. However, in lossy photonic crystals the Floquet-Bloch phase, q, is complex throughout the spectrum and an abrupt distinction between allowed "band" modes, and forbidden "gap" modes cannot be made [25, 27]. Typically, forbidden propagation regions in lossy photonic crystals are characterized by a quick transition to ultra-high $\operatorname{Im}(q)$ values [25].

The fields in the left and right hand side of Eq. (20) can be related by the transfer matrix of the elementary building block, $M^{B B}$, which is equal to $M^{21} P^{1} M^{12} P^{2}$. Thus, $e^{-i q a}$ should be an

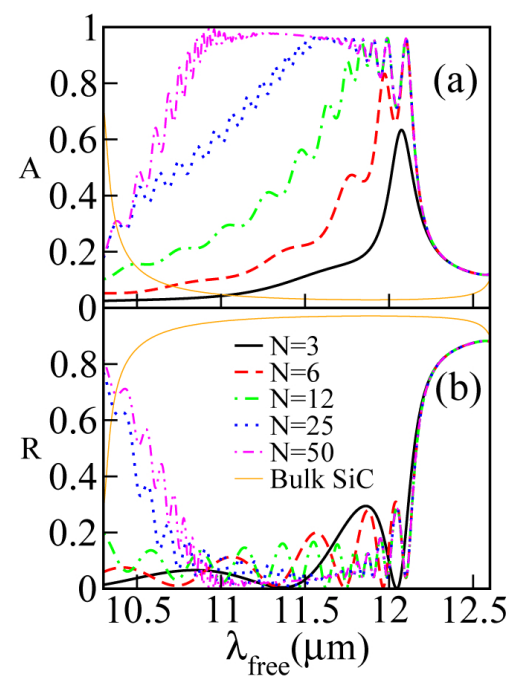

FIG. 5 (a) Absorptance A, versus free space wavelength, $\lambda_{\text {free }}$, for the aperiodic PC of Figure $1(\mathrm{a})$. We show the results for $\mathrm{N}=3$ (black solid), 6 (red dashed), 12 (green dot-dashed), 25 (blue dotted) and 50 (magenta dot-dashed-dot) building blocks. (b) same as (a), but for reflectance $R$, versus free space wavelength. For comparison we also show the absorptance and reflectance from a bulk SiC block with solid-orange lines.

eigenvalue of the elementary building block transfer matrix, which yields:

$$
e^{-\mathrm{iqa}}=\frac{1}{2}\left(M_{11}^{B B}+M_{22}^{B B} \pm \sqrt{\Delta}\right),
$$

with $\Delta=\left(M_{11}^{B B}+M_{22}^{B B}\right)^{2}-4 \operatorname{det}\left(M^{B B}\right)$ and $\operatorname{det}\left(M^{B B}\right)$ being the determinant of the $M^{B B}$ matrix. Eq. (21) yields two possible roots for $q$, with only one being acceptable that satisfies the requirement for passivity. As we are looking for modes propagating in the $+x$-direction the passivity requirement takes the form of $\operatorname{Im}(q)>0[44,45]$. Note that the matrix elements of the $M^{B B}$ matrix are frequency dependent, due to the frequency dependent permittivity of $\mathrm{SiC}$, as given in Eq. (1). Solving Eq. (21) is equivalent to obtaining $q(\omega)$, i.e. the the band structure of the periodic stack.

\section{BROADBAND NEAR-PERFECT ABSORPTANCE WITH APERIODIC SIC PCS IN THE RESTSTRAHLEN BAND: RESULTS AND DISCUSSION}

In this section, we present our results for the absorption of the aperiodic PCs of Section 2, calculated with the TMM method as we described above. In particular, in Figures 5(a) and 5(b) we show the respective spectral response of absorptance and reflectance of the first type of aperiodic PC depicted in Figure 1(a). We observe in Figure 5(a) a near-perfect absorptance when we take 50 building blocks that covers most of the Reststrahlen band (dot-dash-dot magenta line in Figure 5(a)). This is related to the broadband low reflectance seen in Figure 5(b). Conversely, we present the corresponding results of absorptance and reflectance for the aperiodic PC of Figure 1(b) in Figures 6(a) and 6(b) respectively.

Although we successfully demonstrated the broadband ab- 


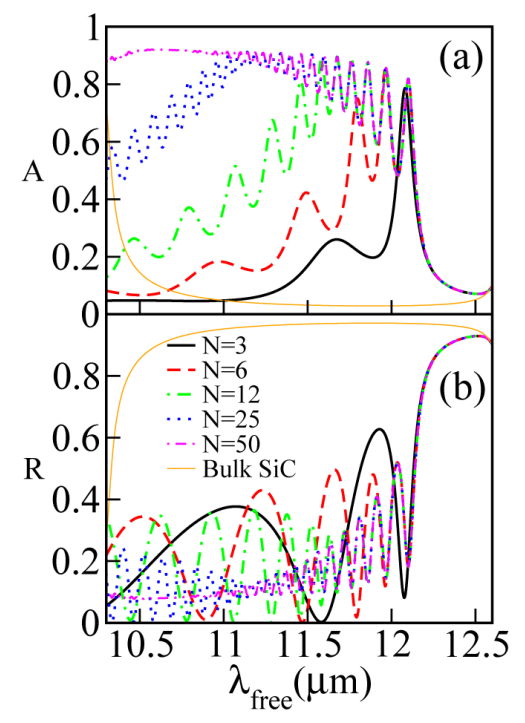

FIG. 6 Same as in Figure 5, but for the aperiodic PC of Figure 1(b)

sorptance with the two aperiodic PCs with large number of building blocks, it is interesting to check to which extent such broadband absorptance survives when a smaller number of building blocks are taken. For this purpose, we show in the same figures, the absorptance and reflectance for a lower total number of building blocks, $\mathrm{N}$.

In particular, we take $\mathrm{N}=3,6,12$ and 25 and show the results in Figures 5 and 6 for the respective cases of Figure 1(a) and Figure 1(b), as black-solid line, red-dashed line, green dotdashed line and blue-dotted line, respectively. We observe that the low reflectance property remains reasonably broadband even with a small number of building blocks. This is especially true for the first type of aperiodic PC. In the second type we see much stronger Fabry-Perot oscillations in the reflectance spectrum. This is because in the latter PC, the filling ratio increases quite rapidly from one building block to the next one as we have seen in Figure 2(d). The results of Ref. [24] suggest that the lower the filling ratio of a PC is, the less the reflection is. Actually, in the same work it was shown that the higher filling ratio PC structures are essentially approaching the bulk $\mathrm{SiC}$ behaviour [24]. The above findings imply that the low reflectance merit of the aperiodic PC of Figure 1(a) can be attributed to the low filling ratio. Such low reflectance with the small strength of Fabry-Perot oscillations is important for a smoother spectral response of the absorption. Obviously, any reflection peaks inadvertently will manifest as dips in the absorption spectrum which are undesirable.

In order to obtain a quantitative measure of the performance of the aperiodic PC schemes, we estimate the cumulative absorptance enhancement $\mathrm{CA}_{\text {enhar }}$ across the Reststrahlen band as follows:

$$
\mathrm{CA}_{\text {enha }}=\frac{1}{\lambda_{2}-\lambda_{1}} \int_{\lambda_{1}}^{\lambda_{2}} \frac{\mathrm{A}_{\mathrm{PC}}(\lambda)}{\mathrm{A}_{\mathrm{SiC}}(\lambda)} d \lambda
$$

where $\lambda_{1}$ and $\lambda_{2}$ represent the bounds of the Reststrahlen band of $\mathrm{SiC}$, and $\mathrm{A}_{\mathrm{PC}}(\lambda)$ and $\mathrm{A}_{\mathrm{SiC}}(\lambda)$ are the respective absorptance of the aperiodic PC and a $200 \mu \mathrm{m}$ thick block of $\mathrm{SiC}$ at the specified wavelength. We show the results in Figure 7(a) for the aperiodic PCs of Figure 1(a) and Figure 1(b)

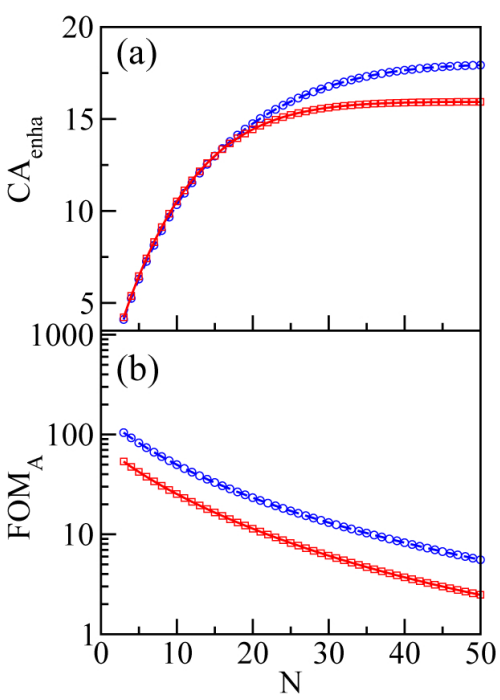

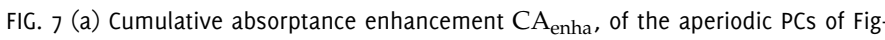
ures 1 (a) (blue circles) and 1 (b) (red squares) versus the total number of building blocks N. (b) Same as (a) but for the absorptance figure of merit $\mathrm{FOM}_{\mathrm{A}}$.

indicated with blue circles and red squares respectively. We can notice that both the aperiodic PCs have almost same cumulative absorptance enhancement for less than 20 building blocks. However when the number of building blocks exceeds 20, we can clearly observe that the first type of aperiodic PC exhibits a higher cumulative absorptance enhancement. We attribute this result to the better reflectance performance of the first type of aperiodic PC due to the consistently lower filling ratio throughout the aperiodic PC as we discussed.

It is also interesting to see which of the two aperiodic PC schemes exhibits a high cumulative absorptance with less absorbing material. Therefore we introduce an absorptance figure of merit $\mathrm{FOM}_{\mathrm{A}}$, as follows:

$$
\mathrm{FOM}_{\mathrm{A}}=\mathrm{CA}_{\text {enha }} \frac{\left(\lambda_{1}+\lambda_{2}\right)}{2 \cdot \mathrm{d}_{\text {thick }}},
$$

where $\mathrm{d}_{\text {thick }}$ is the total thickness of the $\mathrm{SiC}$ in the entire aperiodic PC structure and $\lambda_{1}, \lambda_{2}$, and $\mathrm{CA}_{\text {enha }}$ have the same meaning as in Eq. (22). We plot the $\mathrm{FOM}_{\mathrm{A}}$ in Figure 7(b) for the aperiodic PCs of Figure 1(a) and Figure 1(b) indicated with blue circles and red squares respectively. We can clearly identify the advantage of the first aperiodic PC scheme where a higher $\mathrm{FOM}_{\mathrm{A}}$ is achieved consistently with the same number of building blocks.

We note in passing that we did explore another type of aperiodic PC in which the lattice constant changed linearly, while keeping the filling ratio constant. We explored this route and found that the aforementioned aperiodic PC does not perform any better than the aperiodic PCs of Figure 1(a) and Figure $1(b)$ that we discussed above. We attribute this result to the large Fabry-Perot reflections arising from the increased air layers thickness between the absorbing SiC layers. Accordingly, we did not pursue this scheme further, and we will focus only on the schemes of Figure 1(a) and Figure 1(b) from now on. 


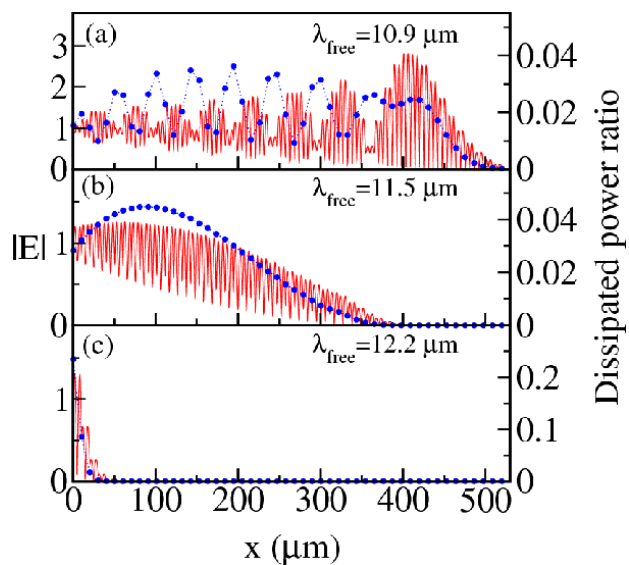

FIC. 8 Spatial electric-field distributions (red solid lines) and dissipated to incident power ratio in the individual absorbing layers (blue circles) versus the location $x$, within the aperiodic PC of Figure 1(a), with 50 building blocks. Results for these are plotted at three free space wavelengths, $\lambda_{\text {free }}$ as indicated in the above individual panels (a), (b) and (c).

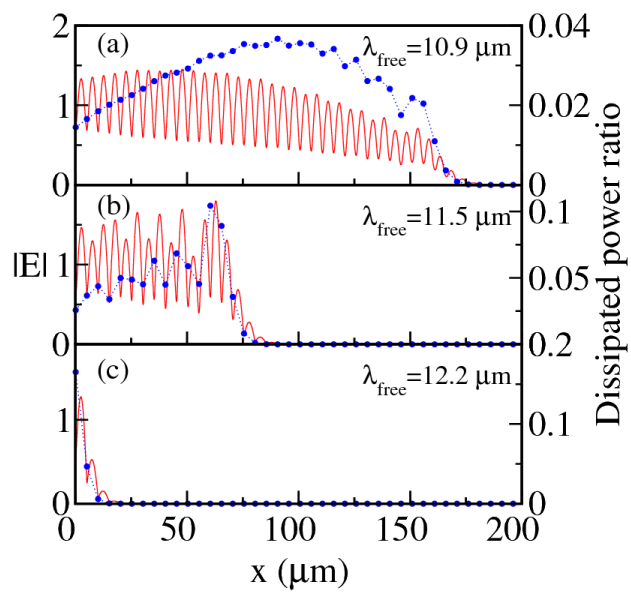

FIC. 9 Same as in Figure 8, but for the aperiodic PC of Figure 1(b). First 40 building blocks are zoomed.

To gain more insight into the performance of the two aperiodic PCs, we also calculate the spatial electric-field distributions inside the two aperiodic PC structures and indicate the results with red-solid lines in Figures 8 and 9 respectively. The panels (a), (b) and (c) in both Figures 8 and 9, represent wavelengths around the blue edge, middle and red edge of the $\mathrm{SiC}$ Reststrahlen band. We can notice from Figures 8 and 9, that around the blue edge of the $\mathrm{SiC}$ Reststrahlen, electric fields are oscillating across the aperiodic PC structure and attenuated nearly at the end of the aperiodic PC. On the other hand, around the middle wavelength of the $\mathrm{SiC}$ Reststrahlen band, the oscillating electric fields are slowly decreasing and strongly attenuated when reaching the middle of the PC. Finally, around the red edge of the SiC Reststrahlen band, the electric field is rapidly attenuated within the first few building blocks of the aperiodic PC.

In order to understand, how these electric-field variations correlate to the overall absorption, we evaluate the ratio of dissipated to incident power within the SiC layers. From Poynting's theorem [46] it can be shown that such dissipated to incident power ratio for the $\mathrm{j}^{\text {th }}$ layer inside the aperiodic PC is
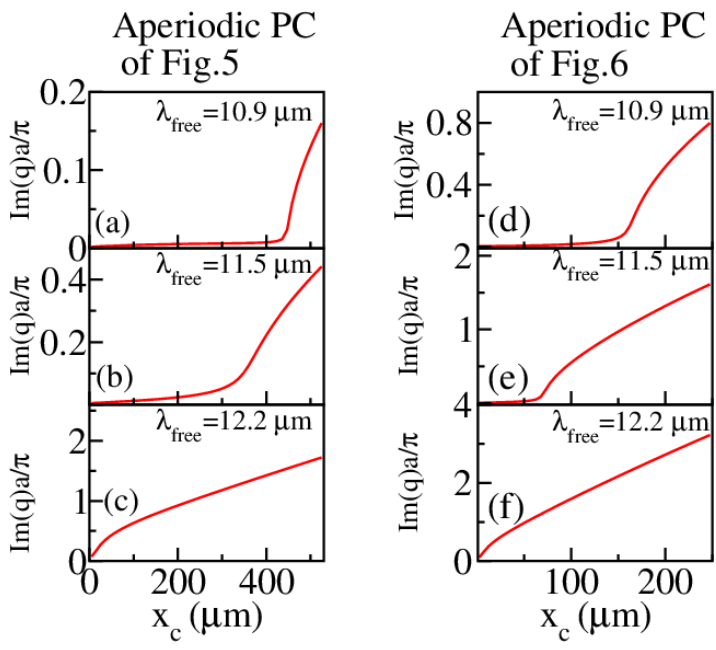

FIG. 10 Band structure properties of the aperiodic PC building blocks versus the location $x_{c}$ of their respective centres. The imaginary part of the Floquet-Bloch phase of the corresponding periodic structure, $\operatorname{Im}(q)$, is plotted at a certain free space wavelength that is designated inside each panel. $\operatorname{Im}(\mathrm{q})$ is scaled with $\mathrm{a} / \pi$ with a being the respective building block size. The panels in the left $[(a),(b)$, and $(c)]$ represent the case of the aperiodic PC of Figure 5. Conversely, the panels in the right $[(d),(e)$, and (f)] represent the case of the aperiodic PC of Figure 6.

given by:

$$
\mathrm{P}(\mathrm{j})=\frac{\omega \varepsilon_{1}^{\prime \prime}}{c\left|E_{0}\right|^{2}} \int_{S_{\mathrm{a}}(\mathrm{j})}^{S_{\mathrm{a}}(\mathrm{j})+\mathrm{d}_{1}(\mathrm{j})}|E(x)|^{2} d x,
$$

where $\varepsilon_{1}^{\prime \prime}$ represents the imaginary part of $\varepsilon_{1}$. With the use of the expressions for the electric-field distributions $E(x)$ within the $\mathrm{SiC}$ layers given by Eq. (15), we obtain:

$$
\begin{aligned}
\mathrm{P}(\mathrm{j})=\frac{\omega \varepsilon_{1}^{\prime \prime}}{c\left|E_{0}\right|^{2}}[ & \frac{|A(\mathrm{j})|^{2}}{2 k_{1 x}{ }^{\prime \prime}}\left(1-e^{-2 k_{1 x}^{\prime \prime} \mathrm{d}_{1}(\mathrm{j})}\right) \\
& +\frac{|B(\mathrm{j})|^{2}}{2 k_{1 x^{\prime \prime}}}\left(e^{2 k_{1 x}^{\prime \prime} \mathrm{d}_{1}(\mathrm{j})}-1\right) \\
& \left.+2 \operatorname{Re}\left[\frac{A(\mathrm{j}) B^{*}(\mathrm{j})}{2 i k_{1 x}^{\prime}}\left(e^{2 i k_{1 x}^{\prime} \mathrm{d}_{1}(\mathrm{j})}-1\right)\right]\right],
\end{aligned}
$$

where $k_{1 x}{ }^{\prime}$ and $k_{1 x}{ }^{\prime \prime}$ are the real and imaginary parts of the wavevector $k_{1 x}$, inside $\mathrm{SiC}$. The $A(\mathrm{j})$ and $B(\mathrm{j})$ coefficients in the above equation are calculated from Eqs. (16)-(19).

We plot the results calculated from Eq. (25), for the power dissipation ratio within the $j^{\text {th }} \mathrm{SiC}$ layer versus the position of the center of this layer, $-x_{\mathrm{c}}(\mathrm{j})=S_{\mathrm{a}}(\mathrm{j})+\mathrm{d}_{1}(\mathrm{j}) / 2-$, with the blue circles in Figure 8 for the aperiodic PC of Figure 1(a). Conversely, we show the respective result for the aperiodic PC of Figure 1(b) in Figure 9. It is interesting to notice in Figures 8 and 9 , that the power dissipation ratio variation does not follow the electric-field variations. This at first seems rather odd. However, we should keep in mind that the power dissipation ratio applies only to the $\mathrm{SiC}$ layers. On the other hand, electric fields are plotted for both the $\mathrm{SiC}$ and air layers. Moreover, the $\mathrm{SiC}$ layer thickness increases from one building to the next one throughout the structure. Therefore, thick SiC layers at the far end of the aperiodic PC will absorb more light due to the larger light-matter interaction length for the same electric- 

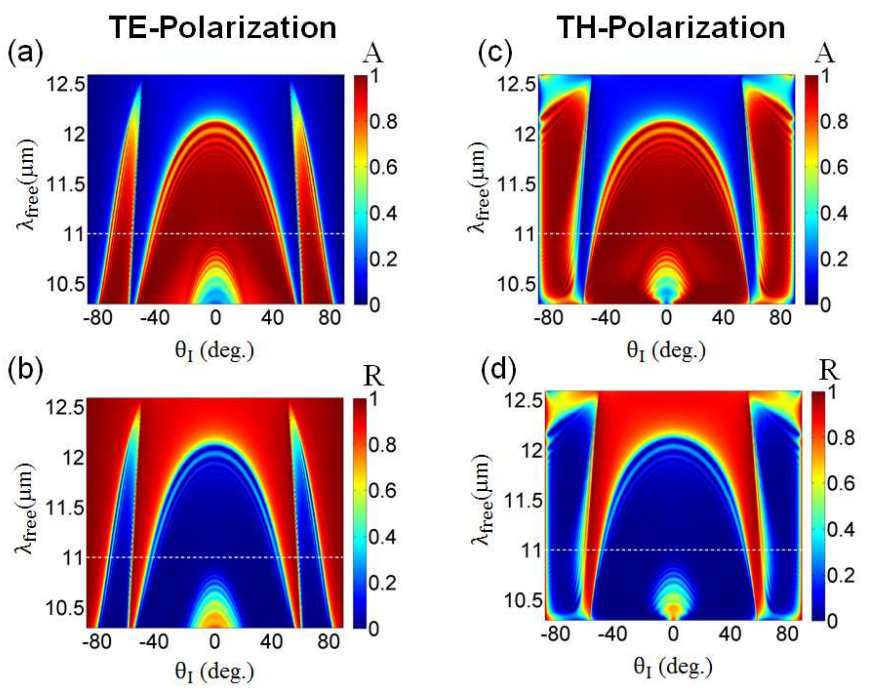

FIC. 11 Angular response of the aperiodic PC of Figure 5. The absorptance, A and reflectance, $R$ are shown versus the impinging's wave angle of incidence $\theta_{I}$ and wavelength $\lambda_{\text {free }}$ for both polarisations. The results in (a) and (b) represent absorptance and reflectance for the TE-polarisation case while the results in (c) and (d) represent absorptance and reflectance for the TH-polarisation case.

field amplitudes in comparison to the thin SiC layers at the front end of the aperiodic PC.

We can notice from Figures 8 and 9 that for the wavelengths around the blue edge of the Reststrahlen band, power is dissipated throughout the aperiodic PC. For wavelengths in the middle of the Reststrahlen band, power dissipation is high at the front end of the PC. For the wavelengths around the red edge of the Reststrahlen band, most of the power is dissipated within the first few SiC layers of the aperiodic PC. These results explain why the presence of a large number of building blocks is more important for the blue side of the Reststrahlen band spectrum, as we have seen in Figures 5 and 6.

We assert that there is a strong correlation between the location of where most power is getting absorbed and the propagation properties of the corresponding building block at such location. Such propagation properties can be characterised by the imaginary part of the Bloch phase $q, \operatorname{Im}(q)$, of the corresponding periodic PC. The latter would signify regions of "forbidden" and "allowed" EM propagation, although such distinction is not as sharp in the case of lossless PCs [25, 27]. In order to uncover such a correlation we calculate the $\operatorname{Im}(q)$ versus $x_{c}$,-for the same wavelength values of the impinging EM wave as in Figs. 8 and 9, for the case of periodic PCs that correspond to the building blocks located at the $x_{c}$ position.

We plot the results, in Figures 10(a), 10(b) and 10(c) at these specified wavelengths (indicated within each panel) for the aperiodic PC of Figure 5. Conversely, in Figures 10(d), 10(e) and $10(\mathrm{f})$ we plot the corresponding results at the same wavelengths for the aperiodic PC of Figure 6. Note that $\operatorname{Im}(q)$ is shown scaled with $\mathrm{a} / \pi$, with $\mathrm{a}=\mathrm{d}_{1}+\mathrm{d}_{2}$ being the building block size, which is constant and equal to 5 microns for the PC of Figure 6 but varies with building block location for the case of Figure 5. Clearly, the results in Figure 10 suggest that at a certain free-space wavelength most power is getting absorbed
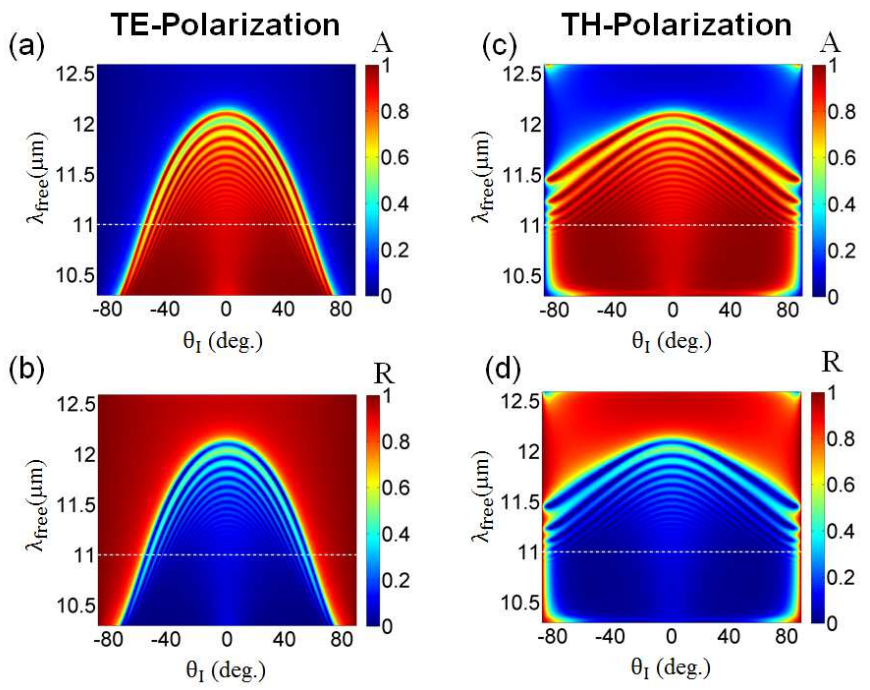

FIG. 12 Same as in Figure 11 but for the aperiodic PC of Figure 6 .

at the vicinity of building blocks with high $\operatorname{Im}(q)$ values, i.e. in the vicinity of the gap regime of the corresponding periodic PCs.

\section{ANGULAR ROBUSTNESS OF THE BROADBAND SUPERABSORPTION}

Realistic beams are finite in lateral extend; they are not plane waves. For example a diffraction-limited beam with a Gaussian lateral profile would be equivalent to a superposition of impinging waves at different incident angles, $\theta_{I}$, within a range spanning roughly between $-10^{0}$ to $10^{0}$. It is therefore interesting to check the robustness of the superabsorptance bandwidth we observed for normal incidence with a plane wave under angular illumination conditions.

While the two polarisations are degenerate for normal incidence they are not so for the case of angular incidence. For this reason we explore the angular absorptance response of our designs under both TE- and TH- illumination conditions that we depicted in Figure 3. We show in Figures 11(a) and 11 (b) respectively the absorptance and reflectance versus incident angle and free space wavelength for a TE-polarised impinging wave on the aperiodic PC structure of Figure 5. The corresponding results for the case of a $\mathrm{TH}$-polarised wave are shown in Figures 11(c) and 11(d). The results were calculated with the TMM method, as we described in the methodology section for the case of a general incidence with $\theta_{I} \neq 0$. Likewise, we also investigate off-normal incidence for the PC of Figure 6 and show our results in an analogous manner in Figure 12.

All cases demonstrate an impressive angular bandwidth throughout the phonon-polariton spectrum that well exceeds the angle-span of a diffraction-limited Gaussian beam. These results suggest that our proposed aperiodic PC platforms would be entirely functional under realistic illumination conditions. 

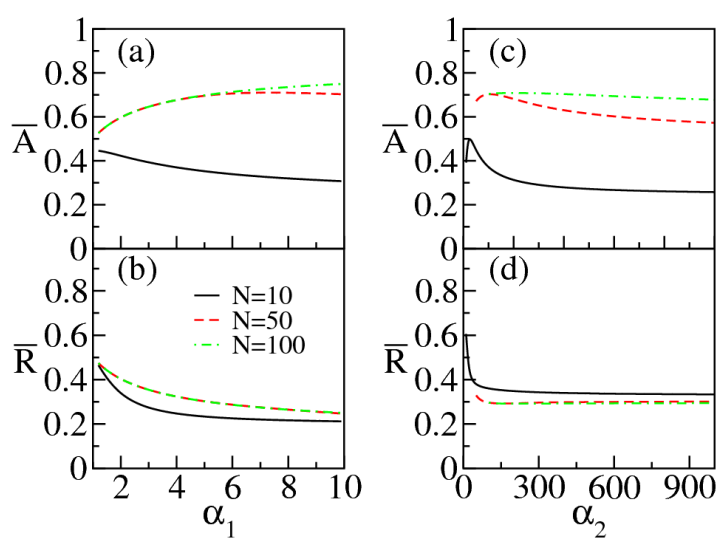

FIC. 13 Aperiodic design performance versus degree of adiabaticity, $\alpha_{1}$, for the aperiodic lamellar sequencing of Eq. (28) [panels (a) and (b)] and versus $\alpha_{2}$ for the aperiodic lamellar sequencing of Eq. (29) [panels (c) and (d)]. The remaining parameters are the same as in the respective cases of Figures 5 and 6 . Both the averaged absorptance, $\overline{\mathrm{A}}$, and the averaged reflectance, $\overline{\mathrm{R}}$ over the entire Reststrahlen band of $\mathrm{SiC}$ are shown.

\section{BROADBAND APERIODIC SUPERABSORBING PCS: DESIGN OPERATION AND OPTIMISATION}

In the previous section we considered two particular aperiodic PC designs. We constructed these two designs based on the intuition we obtained from the study of corresponding periodic structures [24, 25]. We considered for the first building block such parameters that give a low filling ratio and a total size equal to the lattice constant of some of the high performing PCs that were previously investigated [25]. In fact, the first building block of our second aperiodic PC design is exactly the same as one of such periodic designs reported in Ref. [25]. Subsequently, we construct the aperiodic sequence of lamellae with two different scenarios -linearly increasing SiC slab thickness with air thickness kept constant and linearly increasing filling ratio with building block size being kept constant. Again, the linear sequencing slope parameters, $1 / 5$ for the first scenario and 1/100 in the second, were chosen intuitively so that they would produce an adiabatic, yet reasonably significant, increase in the $\mathrm{SiC}$ slabs thickness.

In this section we investigate further the broadband merit of such aperiodic polaritonic PCs beyond the intuitive construction of the designs of Figures 5 and 6. Our purpose is to gain further understanding on the principles of operation of such structures. Moreover, we will investigate the structural parameter tolerance of the broadband absorptance feature of the aperiodic designs and compare our initial intuitively conceived designs with optimised structures.

Firstly, we study the impact of only the sequencing slope on the broadband absorption merit. As a quantitative measure of the latter we consider the averaged absorption, $\overline{\mathrm{A}}$ over the phonon-polariton regime calculated from:

$$
\overline{\mathrm{A}}=\frac{1}{\lambda_{2}-\lambda_{1}} \int_{\lambda_{1}}^{\lambda_{2}} \mathrm{~A}_{\mathrm{PC}}(\lambda) d \lambda
$$

We will also look at the averaged reflection, $\overline{\mathrm{R}}$, given by:

$$
\overline{\mathrm{R}}=\frac{1}{\lambda_{2}-\lambda_{1}} \int_{\lambda_{1}}^{\lambda_{2}} \mathrm{R}_{\mathrm{PC}}(\lambda) d \lambda .
$$

In the above equations, $\mathrm{A}_{\mathrm{PC}}(\lambda)$ and $\mathrm{R}_{\mathrm{PC}}(\lambda)$ represent respectively the absorptance and reflectance of the aperiodic PC structures at a certain free space wavelength $\lambda$. Also, $\lambda_{1}$ and $\lambda_{2}$ represent the blue and red edge of the phonon-polariton spectrum of $\mathrm{SiC}$.

We essentially start from the same building blocks as in the designs of Figure 5 and 6 and change only the slope of the monotonically increasing functions of Eqs. (2) and (3). In particular we take:

$$
d_{1}(j)=d_{1}(1)+\frac{1}{\alpha_{1}}(j-1) \cdot d_{1}(1),
$$

and

$$
f(j)=f(1)+\frac{1}{\alpha_{2}}(j-1),
$$

with $d_{1}(1)=0.125 \mu \mathrm{m}$, and $\mathrm{f}(1)=0.05$ as in the designs of Figures 5 and 6 . We will refer in the following to $\alpha_{1}$ and $\alpha_{2}$ as adiabatic parameters since they control how adiabatic is the increase in $\mathrm{SiC}$ thickness as we progress within the lamellae sequence. The larger the $\alpha$ parameters are the more adiabatic such change is.

We plot our results for $\overline{\mathrm{A}}$ and $\overline{\mathrm{R}}$ versus the adiabatic parameter $\alpha_{1}$ in Figures 13(a) and 13(b) for the aperiodic sequence of the type of Eq. (28) with the remaining structural parameters taken the same as in Figure 5. Conversely, we show the corresponding results for $\overline{\mathrm{A}}$ and $\overline{\mathrm{R}}$ versus the adiabatic parameter $\alpha_{2}$ in Figure 13(c) and 13(d) for the aperiodic sequence of the type of Eq. (29) with the remaining structural parameters taken the same as in Figure 6. We do so for three different cases of total building blocks, $\mathrm{N}$. We take $\mathrm{N}=10,50$ and 100 and show the corresponding results in Figure 13 with solid black, dashed red and dot-dashed green lines respectively. Note that there is a minimum value for the range of the $\alpha_{2}$ parameter that depends on the total number of building blocks as the filling ratio cannot exceed the value of 1 for any building block.

As our intuition would have expected the averaged reflection, $\overline{\mathrm{R}}$, monotonically decreases with increasing $\alpha$ parameters i.e. with increasing adiabaticity of the aperiodic lamellae sequencing. However, clearly we do not see this behavior for the averaged reflectance to be transferred to the averaged absorptance. A monotonically decreasing $\bar{R}$ should perhaps yield a monotonically increasing $\overline{\mathrm{A}}$ with increasing $\alpha$ values. Indeed, we observe so for the large, hundred-building-block, structures.

However, the other cases typically have a maximum for $\overline{\mathrm{A}}$ at a particular value of $\alpha$ or even show a decreasing behavior of $\overline{\mathrm{A}}$ with increasing $\alpha$ as for example the case of Figure 13(a) for $\mathrm{N}=10$. This is actually not too surprising, as transmission maybe non-zero for the thinner structures. As the degree of adiabaticity becomes larger, a larger number of building blocks are required to completely seize the EM energy passing through.

Evidently, as $\alpha_{1} \rightarrow \infty$ and $\alpha_{2} \rightarrow \infty$ the aperiodic lamellar sequences of Eq. (28) and (29) fall back to a periodic pho- 


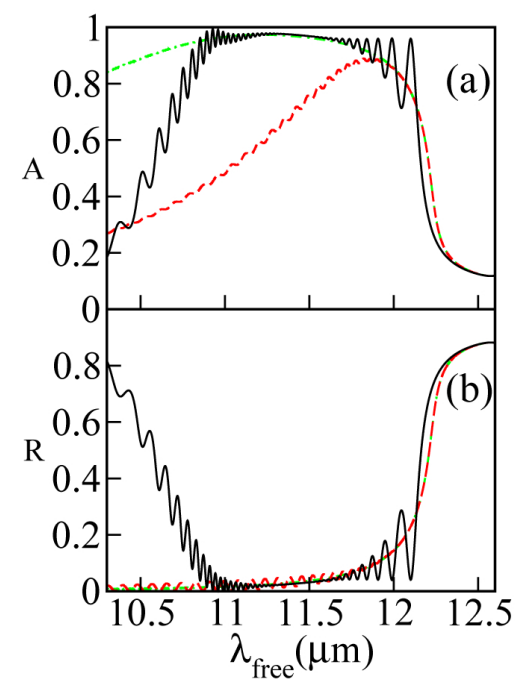

FIG. 14 Comparison of the absorptance spectra [in (a)] and reflectance spectra [in (b)] between the cases of the aperiodic PC of Figure 5 with 50 building block (black solid lines) and its periodic counterpart constructed from the entry building block. The reddashed lines represent the result for the periodic PC with 50 building blocks while the green dot-dashed lines represents the result for the periodic PC with 295 building blocks which corresponds to the same total SiC thickness as the aperiodic structure.

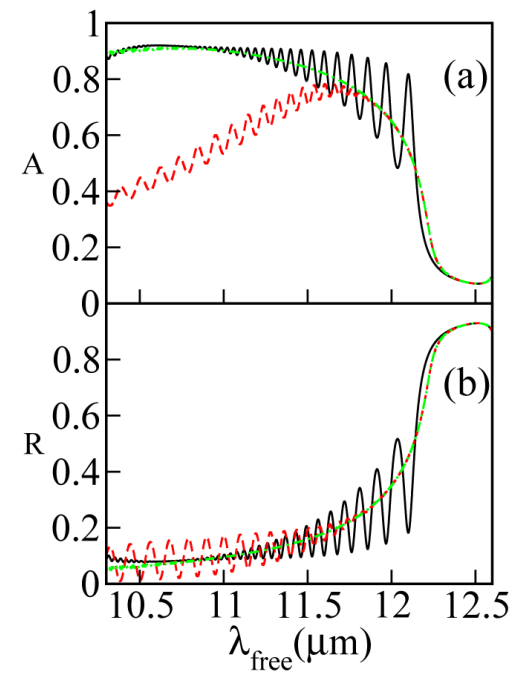

FIG. 15 Same as in Figure 14 but for the case of the aperiodic PC of Figure 6 and the corresponding periodic PC counterparts.

tonic crystal having an elementary unit cell the same as the first building block of these sequences. The asymptotic behavior of the averaged absorptance versus the adiabatic parameters then suggests that these corresponding periodic photonic crystal structures should be capable of a broadband lowreflective and high-absorbing behavior. However, we had not observed that in our prior investigations in Refs. [24] and [25]. This has prompted us to look into this further.

We replot in Figure 14 the absorptance and reflectance versus the free space wavelength for the aperiodic PC of Figure 5 for fifty building blocks as black solid lines to compare it with the corresponding periodic PC constructed from the first building block of the aperiodic structure (shown as red-dashed lines in the same figures). We will refer to such structure simply as
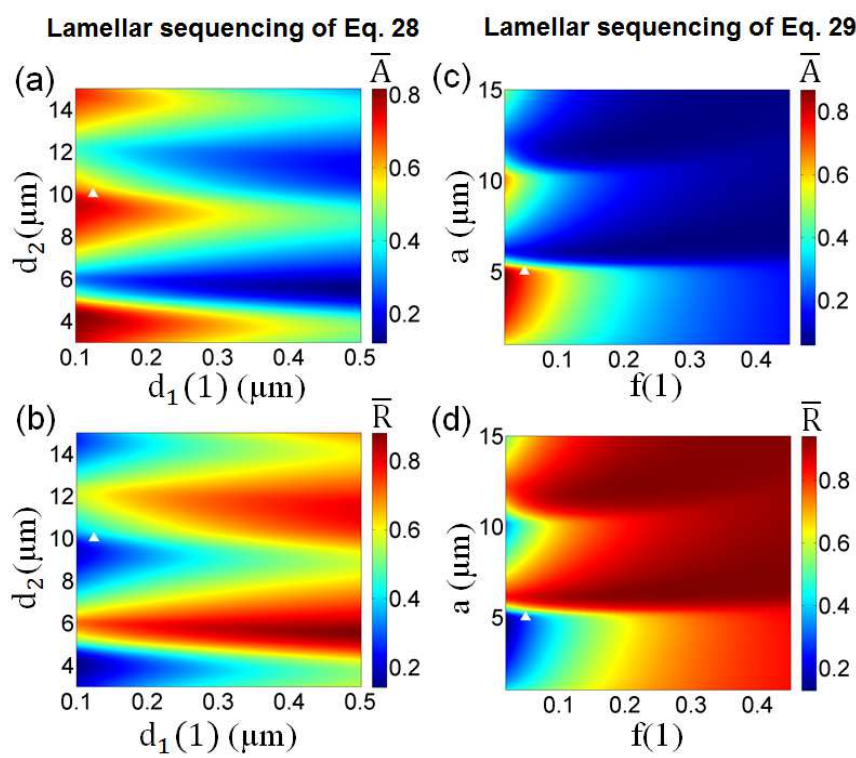

FIC. 16 (a) Spectrally averaged absorptance, $\bar{A}$, of the aperiodic lamellar sequence of Eq. (28) versus thickness of the entry lamella, $d_{1}(1)$, and inter-lammelar spacing, $\mathrm{d}_{2}$, kept constant throughout the sequence. The adiabaticity, $\alpha_{1}$, equals to 5 . (b) Same as in (a) but for the spectrally averaged reflectance, $\bar{R}$. (c) Spectrally averaged absorptance, $\bar{A}$, of the aperiodic lamellar sequence of Eq. (29) versus filling ratio of the entry building block, $\mathrm{f}(1)$, and unit cell size, a, kept constant constant throughout the sequence. The adiabaticity, $\alpha_{2}$, equals to 100. (d) same as in (c) but for the spectrally averaged reflectance $\bar{R}$. The white triangles designate the parameters of the respective aperiodic PCS of Figures 5 and 6.

the "corresponding periodic $\mathrm{PC}^{\prime}$ from hereon. These results clearly manifest that fifty building blocks sequenced according to Eq. (28) for $\alpha_{1}=5$ seize almost all impinging EM energy in most of the spectral range within the Reststrahlen band. Nevertheless, fifty identical building blocks with the same parameters as the first building block of the aperiodic sequence do not. The result is even worse for periodic structures with a unit cell corresponding to any other building from the aperiodic lamellar structure.

Yet, the analysis of Figure 13 suggests that the corresponding periodic PC should possess broadband near-perfect absorptance as well. We do in fact notice a broadband low reflectance in Figure 14(b). This clearly points out that the lack of absorptance bandwidth in the corresponding periodic $\mathrm{PC}$ is because of insufficient $\mathrm{SiC}$ material in the fifty-cell structure. Thus, we subsequently consider a corresponding periodic PC that would have as much $\mathrm{SiC}$ material as the aperiodic structure of fifty building blocks, N. This means that such periodic PC would need to have a $N^{e q}$ number of unit cells given by:

$$
N^{e q}=N+\frac{1}{10} N(N-1)
$$

where $N=50$, i.e. it should be comprised of 295 unit cells. We show the corresponding spectra for this long periodic PC in Figure 14 with green dot-dashed lines. Indeed, a broadband absorptance behavior is now observed.

We make a similar comparison in Figure 15 for the corresponding periodic PC case of the aperiodic lamellar sequence of Eq. (29), with the parameters of Figure 6. We show the results for the absorptance and reflectance for this aperiodic PC 


\begin{tabular}{|ccccccc|}
\hline $\begin{array}{c}\text { Type of } \\
\text { Lamellar Structure }\end{array}$ & $\begin{array}{c}\text { Building } \\
\text { Block No }\end{array}$ & $\begin{array}{c}\text { Total structure } \\
\text { thickness }(\mu \mathbf{m})\end{array}$ & $\begin{array}{c}\text { SiC } \\
\text { thickness }(\mu \mathbf{m})\end{array}$ & $\mathbf{F O M}_{\mathbf{A}}$ & $\mathbf{F O M}_{\mathbf{A}}{ }^{\prime}$ & $\mathbf{C A}_{\mathbf{e n h a}}$ \\
\hline Aperiodic PC of Figure 5 & 50 & 530.625 & 36.875 & 5.56 & 0.387 & 17.92 \\
\hline Corresponding periodic PC & 50 & 500 & 6.25 & 26.12 & 0.326 & 14.26 \\
\hline Corresponding periodic PC & 295 & 2950 & 36.875 & 5.80 & 0.073 & 18.69 \\
\hline \hline Aperiodic PC of Figure 6 & 50 & 250 & 73.75 & 2.47 & 0.730 & 15.93 \\
\hline Corresponding periodic PC & 50 & 250 & 12.5 & 11.94 & 0.597 & 13.03 \\
\hline Corresponding periodic PC & 295 & 1475 & 73.75 & 2.46 & 0.123 & 15.85 \\
\hline
\end{tabular}

TABLE 1 Performance evaluation and comparison of the aperiodic PCS of Figures 5 and 6 and their periodic counterparts constructed from their entry building block. The absorptance figure of merits, $\mathrm{FOM}_{\mathrm{A}}$ and $\mathrm{FOM}_{\mathrm{A}}{ }^{\prime}$ as defined in Eqs. (23) and (32) respectively, as well as the cumulative enhancement, CA $\mathrm{C}_{\mathrm{enha}}$ as defined in Eq. (22) are employed for such purpose.

Lamellar sequencing of Eq. 28

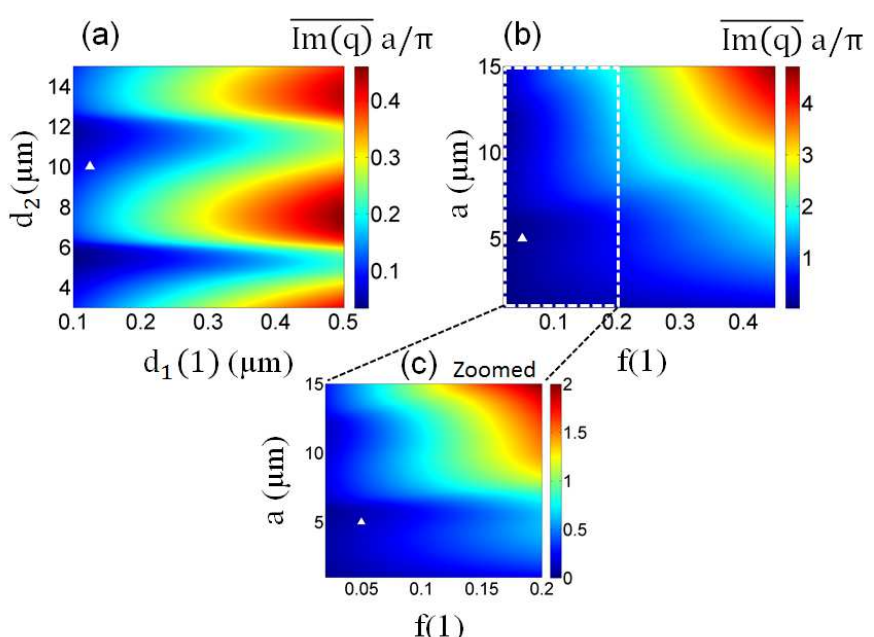

FIG. 17 Same as in Figures $16(\mathrm{a})$ and $16(\mathrm{c})$ but for $\overline{\operatorname{Im}(\mathrm{q})}$ evaluated from Eq. (33). The $\overline{\operatorname{Im}(q)}$ is scaled with $a / \pi$, with a being the lattice constant of the corresponding periodic PC. For the case in (a) a $=d_{1}(1)+d_{2}$. For the case in (b) a is explicitly given in the vertical axis.

and its periodic counterpart with $\mathrm{N}=50$ buildings blocks as black solid lines and red-dotted lines respectively. As in the case of Figure 14 we also see here that fifty cells are inadequate to seize the incoming EM energy over a broad bandwidth in the Reststrahlen band for periodic PC structure. The number of $N^{e q}$ unit cells we need here in order to have the same amount of $\mathrm{SiC}$ material in the corresponding periodic $\mathrm{PC}$ as in the aperiodic structure of Figure 6 is given by:

$$
N^{e q}=\frac{1}{f(1)}\left(f(1) N+\frac{1}{200} N(N-1)\right),
$$

with $N=50$ and $f(1)=0.05$, which also is equal to 295 . We plot in Figure 15 the spectra for the corresponding periodic PC with 295 unit cells as green dot-dashed line.

Indeed, the results in Figures 14 and 15 verify that the corresponding periodic PC counterparts exhibit broadband absorptance behavior when a very large number of unit cells is taken that provides a sufficient amount of SiC material. Thus, the above analysis suggests that if we take a block of $\mathrm{SiC}$ material and slice it to equal very thin slices sufficiently spaced apart at equal distances we can obtain efficient broadband absorption in the Reststrahlen band of SiC. Note that the total length $L$ of such periodic structure is really very large. In particular, it is close to 300 free space wavelengths for the case of Figure 14 and close to 150 free space wavelengths for the case of Figure 15. On the other hand, if we allow to slice the same amount of $\mathrm{SiC}$ material in unequal slices and position them so that their thickness gradually increases we can obtain a similar absorption over a broad spectral range with much more compact structures. In particular, the length of the structures in Figure 5 and Figure 6 are close to 50 and 25 wavelengths respectively.

From the practical point of view, it is important to also consider the total length of the structure when evaluating the merits of a certain design. Hence, it becomes then clear that $\mathrm{FOM}_{\mathrm{A}}$, we defined in Eq. (23) may not by itself be an adequate measure of performance of a certain design. We therefore introduce an additional figure of merit, $\mathrm{FOM}_{\mathrm{A}}{ }^{\prime}$ defined as follows:

$$
\mathrm{FOM}_{\mathrm{A}}^{\prime}=\mathrm{CA}_{\mathrm{enha}} \frac{\left(\lambda_{1}+\lambda_{2}\right)}{2 L}
$$

with $L$ being the total thickness of the structure. We emphasize that these figures of merit are useful for a comparative analysis between designs, in the sense that they help to identify the advantages and disadvantages of a certain design. However one should bear in mind that they are always trade-offs to consider in each case.

For example it appears that the periodic structures can yield smooth broad spectra of efficient absorptance but they involve a much higher number of lamellae and are much longer (about six times) than their aperiodic counterparts. The more compact we try to make a broadband design, the thicker SiC blocks we need to introduce which inevitably lead to more prominent Fabry-Perot fringes and less smooth spectra. For example, the aperiodic PC of Figure 5 shows more interference fringes than its ultra-long corresponding periodic PC counterpart but less interference fringes in comparison with the more compact aperiodic PC of Figure 6. Table 1 encapsulates such comparisons, where both introduced absorptance figure of merits, $F O M_{A}$ and $F O M_{A}^{\prime}$, are given for the designs we analysed above.

We proceed below in evaluating further the robustness of the broadband absorptance merit of the aperiodic PCs. Figure 13 attests that the adiabaticity parameter values of $\alpha_{1}=5$ and $\alpha_{2}=100$ are near the optimum values that yield the maximum averaged absorptance, $\overline{\mathrm{A}}$ when fifty building blocks are 
taken in the lamellar sequences of Eqs. (28) and (29) respectively. Accordingly, below we fix these parameters at these values.

Then for the lamellar sequence emanating from Eq. (28), we calculate how the averaged absorptance changes with $\mathrm{d}_{1}(1)$ and inter-SiC lamellae spacing $\mathrm{d}_{2}$ which is kept constant throughout the entire sequence. Conversely, for the lamellar sequence emanating from Eq. (29), we calculate how the averaged absorptance is changing with the filling ratio of the entry building block, $\mathrm{f}(1)$, and the building block size, a, which is kept constant throughout the entire sequence. We plot the results in Figures 16(a) and 16(c) respectively. The accompanying results for the averaged reflectance, $\bar{R}$, are shown in Figures 16(b) and 16(d) respectively.

We observe that there is a wide range in parameter space of near-optimum performance in both cases attesting for the robustness of the designs. This is especially true for the designs emanating from the sequence of Eq. (28). We observe that the performance of both designs deteriorates with increasing filling ratio. The white triangles in the parameter space designate the parameters of the designs considered in Figure 5 and Figure 6. It is impressive to observe that both designs that we conceived intuitively are very close to the optimised aperiodic PC designs.

In the following, we try to understand further the particular structure of the absorptance behavior we observed in Figure 16 in the parameter space of the lamellar sequences. We argue that the performance of the aperiodic PC designs is strongly correlated to the properties of their entry building block. In particular, we argue that the spectral extend of the allowed modes of a periodic PC constructed from the entry building block is of crucial significance. As a measure of the latter we consider the average value of the imaginary part of the Floquet-Bloch phase $q, \overline{\operatorname{Im}(q)}$. The larger this is the lower the extend of allowed modes within the SiC Restrahlen band for the periodic PC comprised of the entry building block of the aperiodic structure. In other words it would be the minima of $\overline{\operatorname{Im}(\mathrm{q})}$ that would suggest a strong absorptance performance.

We evaluate $\overline{\operatorname{Im}(q)}$ from:

$$
\overline{\operatorname{Im}(\mathrm{q})}=\frac{1}{\lambda_{2}-\lambda_{1}} \int_{\lambda_{1}}^{\lambda_{2}} \operatorname{Im}(\mathrm{q}, \lambda) d \lambda,
$$

where $\operatorname{Im}(q, \lambda)$ is calculated with the TMM method as we discussed in the methodology section. The parameter space of Figure 16 defines different entry building blocks and for each we calculate such $\overline{\operatorname{Im}(q)}$ value for the corresponding PC medium. We plot the result in Figure 17(a) for entry building blocks corresponding to the sequencing of Eq. (28). Conversely, we show in Figure 17(b) the result for entry building blocks corresponding to the sequencing of Eq. (29). Figure 17(c) is a zoom of Figure 17(b) around the region of lower filling ratios. It is remarkable to observe that the landscape of $\overline{\operatorname{Im}(q)}$ in the parameter space resembles closely that of the averaged reflectance $\bar{R}$ we observed in Figure 16 .

This strongly supports our claim that the properties of the en- try building block strongly define the broadband absorption performance of the periodic structure provided a proper adiabatic parameter in the lamellar sequence is taken. We stress that the behavior of a certain building block well within the aperiodic sequence can be dramatically different from the corresponding periodic photonic crystal. For example let's take the $41^{\text {th }}$ building block of the aperiodic structure of Figure 5 located at $x_{c} \sim 425 \mu \mathrm{m}$. An EM wave incident with a wavelength of $10.9 \mu \mathrm{m}$ wavelength would fall in the vicinity of the band-gap for the corresponding periodic PC made from such building block. As a result at this wavelength the latter PC is highly reflective, more than $\sim 80 \%$. Yet, at the same impinging wavelength light is directed with a high efficiency to the same building block within the aperiodic sequence to get absorbed thereafter. This further supports our claim that it is the entry unit cell that most crucially influences the absorptance performance of the aperiodic designs.

These observation simultaneously imply that the spatial order of the building blocks of the lamellar sequence would be of crucial significance. We investigate this in detail in the following section.

\section{SIGNIFICANCE OF THE APERIODIC PC'S BUILDING BLOCK SEQUENCE}

We have seen that a large number of absorbing layers are necessary for optimising cumulative absorption enhancement. Both the aperiodic PCs of Figure 1(a) and Figure 1(b) employ these building blocks in an increasing order of thickness. Accordingly, it is natural to wonder how important is the sequence of building blocks in these microstructures. For this reason, we will explore changing the building block sequencing of the aperiodic PC structures and its impact on the absorption spectra.

We start from the case of Figure 1(a) and we just interchange the first two SiC layers of our original aperiodic PC of Figure 1(a). We show the absorptance and reflectance of this modified aperiodic PC structure with the green-dashed lines in Figures 18(a) and 18(b) respectively. We compare these results with those of the original aperiodic PC seen with redsolid lines. We can notice that the absorptance is decreased at the higher wavelength end of the absorptance spectra, corresponding to the converse increase in the reflectance.

Subsequently, we interchange the SiC layers of the first three building block so that original first, second and third layer are placed in the third, first and second building block respectively of the new modified aperiodic PC. We can clearly identify a further dip in the absorptance and converse increase in the reflectance of this PC as indicated with the violet-solid lines in Figures 18(a) and 18(b) respectively. These results suggest that even slightly altering the order of the absorbing layers in the original aperiodic PC negatively affects the cumulative absorptance within the SiC Reststrahlen band.

Now we consider the extreme case where the original building blocks are randomly located. In particular, we apply the Knuth shuffle algorithm [47] on the original aperiodic PC 


\begin{tabular}{|ccccccc|}
\hline $\begin{array}{c}\text { Type of } \\
\text { Lamellar Structure }\end{array}$ & $\begin{array}{c}\text { Building } \\
\text { Block No }\end{array}$ & $\begin{array}{c}\text { Total structure } \\
\text { thickness }(\mu \mathbf{m})\end{array}$ & $\begin{array}{c}\text { SiC } \\
\text { thickness }(\mu \mathbf{m})\end{array}$ & $\mathbf{F O M}_{\mathbf{A}}$ & FOM $_{\mathbf{A}}{ }^{\prime}$ & $\mathbf{C A}_{\text {enha }}$ \\
\hline Aperiodic PC of Figure 20 & 5 & 22.80 & 5.14 & 18.10 & 4.081 & 8.13 \\
\hline Corresponding periodic PC & 5 & 22.80 & 1.140 & 50.52 & 2.526 & 5.03 \\
\hline Corresponding Terminated PC & 5 & 22.686 & 1.026 & 78.79 & 3.563 & 7.06 \\
\hline
\end{tabular}

TABLE 2 Performance evaluation and comparison of the structures of Figure 20. The absorptance figure of merits, $\mathrm{FOM}_{\mathrm{A}}$ and $\mathrm{FOM}_{\mathrm{A}}{ }^{\prime}$ as defined in Eqs. (23) and (32) respectively, as well as the cumulative enhancement, $\mathrm{CA}_{\text {enha }}$ as defined in Eq. (22) are employed for such purpose.

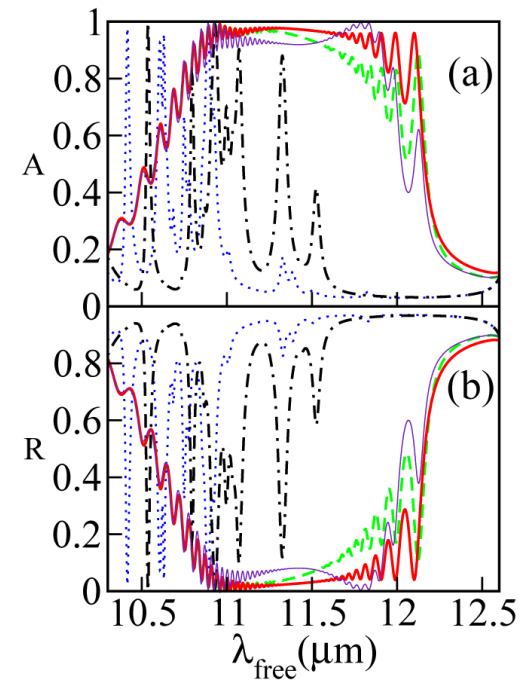

FIC. 18 (a) Spectral response of the absorptance for the modified PCS from the original aperiodic PC of Figure 1 (a). We show the results for the modified PCs in which the first two and the first three SiC layers are interchanged with green-dashed line and violet-solid line respectively. The results for two random multilayer structures, comprised of the same building blocks of the original aperiodic PC of Figure $1(a)$, are represented with blue-dotted line and black dot-dashed line. (b) same as (a), but for the reflectance versus free space wavelength. For comparison, we also show the absorptance and reflectance of the original aperiodic PC of Figure 1(a) with red-solid lines.

building blocks. We show the absorptance of such two random multilayer structures in Figure 18(a), indicated with bluedotted and black dot-dashed lines respectively. The corresponding reflectance is shown in Figure 18(b). We also show the SiC lamellae thickness at each building block with the building block order $j$ for these two random sequences in Figure 19. In panel (a) the case of the blue dotted line of Figure 18 is shown with blue circles. Conversely, in panel (b) the case of the black dot-dashed line of Figure 18 is shown with black circles. In both cases, the corresponding values of the SiC lamellae thickness of the original aperiodic PC are shown for comparison with a red solid line.

We can identify multiple high absorptance peaks in the absorptance spectrum of these random multilayer structures. However, these do not exhibit a broadband absorption. Thus the cumulative absorption is much lower than the one exhibited by their aperiodic PC counterpart. This is because of the high reflectance exhibited by the structures with randomly sequenced building blocks. We carried out the same investigations for the aperiodic PC of Figure 1(b) and we observed similar results. Therefore, our findings suggest that although $\mathrm{SiC}$ layers with different thicknesses absorb best different wave-
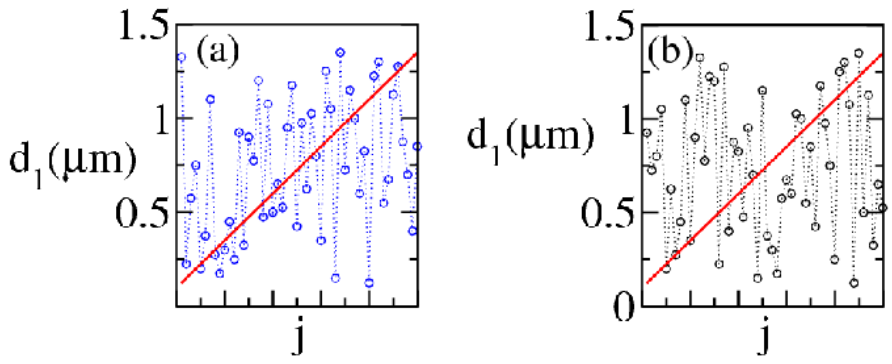

FIG. $19 \mathrm{SiC}$ lamellae thickness in the random sequences versus building order $j$. (a) case of the blue dotted line of Figure 18. (b) case of the black dot-dashed line of Figure 18. In both cases, the corresponding values of the SiC lamellae thickness of the original aperiodic $\mathrm{PC}$ are shown for comparison with a red solid line.

lengths of light, these should be arranged in the increasing order of thickness in order to obtain large cumulative absorption within the entire SiC Reststrahlen band.

\section{SHRINKING THE SIZE OF THE BROADBAND APERIODIC PC SUPERABSORBERS}

Table 1 clearly suggests that the first aperiodic scheme [from Eq. (28)] is better performing with respect to the amount of $\mathrm{SiC}$ material used; hence it is characterised by higher $\mathrm{FOM}_{A}$ values. However, the second aperiodic scheme [from Eq. (29)] performs better with respect to the total structural size. Hence it is characterised by higher $F O M_{A}^{\prime}$ values. We clearly see when we compared the aperiodic PCs of Figures 5 and Figure 6 that the absorption enhancement of the latter saturates quicker with a smaller number of total building blocks [See Figure 7], and performance would not be increased further by adding more building blocks. We see in Figure 9 that for the PC of Figure 6, essentially no more power is getting absorbed after the $35^{\text {th }}$ building block.

In other words, the second aperiodic scheme uses more SiC material, but allows to pack it closer to the entry face yielding a smaller total structural size. On the other hand the first aperiodic scheme uses less $\mathrm{SiC}$ material and disperses it within a larger structural size. In the extreme case of a periodic structure at broadband absorption conditions, we found out in Section 6 that the $\mathrm{SiC}$ material must be very dilute throughout, requiring structures as long $\sim 300$ free space wavelengths. For practical purposes, it is advantageous to pack as much $\mathrm{SiC}$ material as possible to the entry face to obtain compact designs with a fewer number of total building blocks. Yet, that poses a challenge because the more we pack $\mathrm{SiC}$ material close to the entry face the higher the reflectance is. 


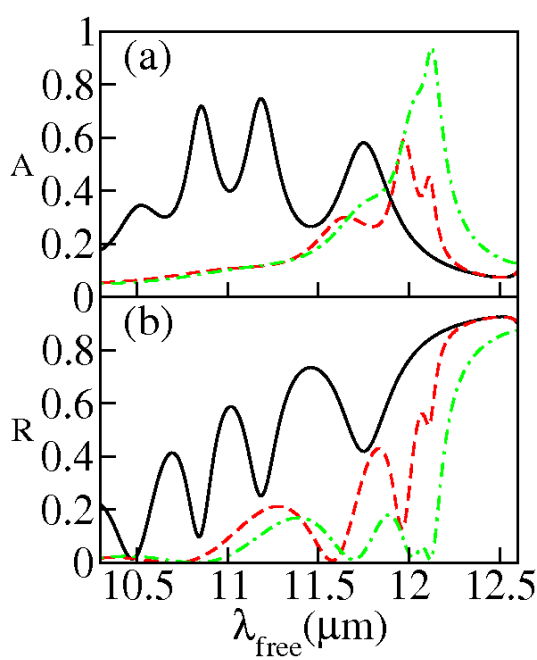

FIC. 20 Two-wavelength long aperiodic PC structure from the lamellar sequence of Eq. (29) with $\mathrm{a}=4.56 \mu \mathrm{m}$ and $\alpha_{2}=11.4$. The absorptance and reflectance are shown with black solid lines in (a) and (b) respectively. For comparison the corresponding spectra of a periodic PC made from the entry building block and with the same total thickness are shown with red long-dashed lines. In addition, we show the effect of a $50 \%$ termination in the entry face of the latter periodic PC with green dot-dashed lines.

We investigate in the following the possibility to obtain a more compact absorber based on the second lamellar sequencing scheme generated from Eq. (29). We would need more SiC material distributed over a smaller number of building blocks which implies the need for a less adiabatic design which unavoidably would be more reflective. We therefore need to relax the requirement of near-perfect absorptance. Thus, we investigate whether it is possible to obtain a good, yet not near-perfect, absorption performance throughout the Reststrahlen band with the lamellar sequence of Eq. (29). We set $\mathrm{a}=4.56 \mu \mathrm{m}$, and $\mathrm{f}(1)=0.05$ which represent near-optimum parameters for the entry building block as we can see in Figure 16. For this parameters we look for the optimum adiabaticity value when a total of five building blocks are considered and we find that to be $\alpha_{2}=11.4$.

We show the results for the absorptance and reflectance versus free space wavelength as black solid lines in Figures 20(a) and 20(b) respectively. We observe a strong absorption performance that spans throughout most of the SiC Reststrahlen band, yielding a cumulative enhancement factor of close to 10 . We clearly see that this aperiodic scheme outperforms the corresponding periodic PC made from the entry building block, -represented with the dashed red lines in the figure. We also compare this performance with the performance of the latter periodic PC with its front face terminated by removing $50 \%$ of $\mathrm{SiC}$ material. We see that the latter optimisation route, that we discussed in detail in Refs. [24, 25], leads to overall comparable absorption enhancement. However, the strong absorption performance of the terminated periodic PC is restricted to a narrow part of the SiC Reststrahlen band. On the other hand, the cumulative absorption enhancement is more evenly distributed across the Reststrahlen band for the aperiodic PC design. We outline the absorption performance comparison between these three designs in Table 2. This underlines the strong potential of the aperiodic PC structures for high performing broadband absorbers.

\section{CONCLUSIONS}

We proposed an aperiodic absorbing PC route to engineering broadband EM absorption. Our results suggest that this approach is promising even when the PC constituent building blocks have a high extinction coefficient and high reflectance, as the case of the SiC paradigm explored here. In particular, we demonstrated a broadband near-perfect absorption with two types of SiC 1D-aperiodic PCs, one in which the thickness of the SiC layer increases linearly, and one in which the filling ratio increases linearly throughout the structure. Our results suggest that the broadband near-perfect absorption merit is tolerant under angular illumination within a span of at least $20^{\circ}$. We found that the first aperiodic PC scheme has better performance in terms of smooth absorption profile and high cumulative absorption enhancement with less $\mathrm{SiC}$ material. On the other hand the second aperiodic PC scheme has better performance in terms of structural size and is promising for broadband superabsorption with designs of the order of the impinging wave's wavelength

\section{ACKNOWLEDGEMENTS}

Financial support for the Ph.D. studentship of G. C. R. Devarapu by the College of Engineering, Mathematics and Physical Sciences (CEMPS) University of Exeter is acknowledged.

\section{References}

[1] H. A. Atwater, and A. Polman, "Plasmonics for improved photovoltaic devices," Nat. Mater. 9, 205-213 (2010).

[2] R. A. Pala, J. White, E. Barnard, J. Liu, and M. L. Brongersma, “Design of Plasmonic Thin-Film Solar Cells with Broadband Absorption Enhancements," Adv. Mater. 21, 3504-3509 (2009).

[3] E. Rephaeli, and S. Fan. "Absorber and emitter for solar thermo-photovoltaic systems to achieve efficiency exceeding the Shockley-Queisser limit," Opt. Express 17 15145-15159 (2009).

[4] X. J. Wang, J. D. Flicker, B. J. Lee, W. J. Ready, and Z. M. Zhang, "Visible and near-infrared radiative properties of vertically aligned multi-walled carbon nanotubes," Nanotechnology 20, 215704 (2009).

[5] Y. Guo, and Z. Jacob, "Thermal hyperbolic metamaterials," Opt. Express 21, 15014-15019 (2013).

[6] X. L. Liu, L. P. Wang, and Z. M. Zhang, "Wideband Tunable Omnidirectional Infrared Absorbers Based on Doped-Silicon Nanowire Arrays," J. Heat Transf. 135, 061602 (2013).

[7] J. G. Fleming, S. Y. Lin, I. El-Kady, R. Biswas, and K. M. Ho, "Allmetallic three-dimensional photonic crystals with a large infrared bandgap," Nature 417, 52-55 (2002).

[8] T. Maier, and H. Brueckl, "Multispectral microbolometers for the midinfrared," Opt. Lett. 35 3766-3768 (2010).

[9] E. L. Dereniak, and D. G. Crowe, Optical Radiation Detectors (John Wiley and Sons, Hoboken, 2008). 
[10] Handbook of Optics Volume I: Fundamentals, Techniques, and Design, M. Bass, E. W. Van Stryland, D. R. Williams, and W. L. Wolfe, eds. (Second Edition, McGraw-Hill, New York, 1995).

[11] A. V. Barve, S. J. Lee, S. K. Noh, and S. Krishna, "Review of current progress in quantum dot infrared photodetectors," Laser and Photonics Reviews 4, 738-750 (2010).

[12] S. J. Lee, Z. Y. Ku, A. Barve, J. Montoya, W. Y. Jang, S. R. J. Brueck, M. Sundaram, et al., "A monolithically integrated plasmonic infrared quantum dot camera," Nat. Commun. 2, 286 (2011).

[13] C. J. Hill, A. Soibel, S. A. Keo, J. M. Mumolo, D. Z. Ting, and S. D. Gunapala, "Demonstration of large format mid-wavelength infrared focal plane arrays based on superlattice and BIRD detector structures," Infrared. Phys. Techn. 52, 348-352 (2009).

[14] J. A. Mason, S. Smith, and D. Wasserman, "Strong absorption and selective thermal emission from a midinfrared metamaterial," Appl. Phys. Lett. 98, 241105-3 (2011).

[15] J. A. Mason, G. Allen, V. A. Podolskiy, and D. Wasserman, "Strong coupling of molecular and mid-infrared perfect absorber resonances," IEEE Photonic. Tech. L. 24, 31-33 (2012).

[16] N. I. Landy, S. Sajuyigbe , J. J. Mock, D. R. Smith, and W. J. Padilla, "Perfect Metamaterial Absorber," Phys. Rev. Lett. 100, 207402 (2008).

[17] C. Wu, and C. Shvets, "Design of metamaterial surfaces with broadband absorbance," Opt. Lett. 37, 308-310 (2012).

[18] X. Liu, T. Starr, A. F. Starr, and W. J. Padilla, "Infrared spatial and frequency selective metamaterial with near-unity absorbance," Phys. Rev. Lett. 104, 207403 (2010).

[19] Y. Avitzour, Y. A. Urzhumov, and G. Shvets, "Wide-angle infrared absorber based on a negative-index plasmonic metamaterial," Phys. Rev. B 79, 045131 (2008).

[20] G. Veronis, R. W. Dutton, and S. Fan, "Metallic photonic crystals with strong broadband absorption at optical frequencies over wide angular range," J. Appl. Phys. 97, 093104 (2005).

[21] C. Lin, and M. L. Povinelli, "Optical absorption enhancement in silicon nanowire arrays with a large lattice constant for photovoltaic applications," Opt. Express 17, 1937 (2009).

[22] Y. Park, E. Drouard, 0. El-Daif, X. Letartre, P. Viktorovitch, A. Fave, A. Kaminski, M. Lemiti, and C. Seassal, "Absorption enhancement using photonic crystals for silicon thin film solar cells," Opt. Express 17, 14312 (2009).

[23] S. Y. Lin, J. G. Fleming, Z. Y. Li, I. El-Kady, R. Biswas, and K. M. Ho, "Origin of absorption enhancement in a tungsten, threedimensional photonic crystal," J. Opt. Soc. Am. B. 20, 1538-1541 (2003).

[24] G. C. R. Devarapu, and S. Foteinopoulou, "Mid-IR near-perfect absorption with a SiC photonic crystal with angle-controlled polarization selectivity," Opt. Express 20, 13040-13054 (2012).

[25] G. C. R. Devarapu, and S. Foteinopoulou, "Compact photoniccrystal superabsorbers from strongly absorbing media," J. Appl. Phys. 114, 033504 (2013).

[26] C. Kittel, Introduction to Solid State Physics (John Wiley and Sons, Hoboken, 2005).

[27] C. Engstrom, C. Hafner, and K. Schmidt, "Computations of lossy bloch waves in two-dimensional photonic crystals," J. Comput. Theor. Nanos. 6, 1-9 (2009).

[28] A. Katzir, A. C. Livanos, J. B. Shellan, and A. Yariv, "Chirped gratings in integrated optics," IEEE J. Quantum. Elect. 13, 296-304 (1977).
[29] A. Mouldi, and M. Kanzari, "Design of an omnidirectional mirror using one dimensional photonic crystal with graded geometric layers thicknesses," Optik 123, 125-131 (2012).

[30] A. Mouldi, and M. Kanzari, "Broad multilayer antireflection coating by apodized and chirped photonic crystal," Opt. Commun. 284 4124-4128 (2011).

[31] P. B. Catrysse, and S. Fan, "Near-complete transmission through subwavelength hole arrays in phonon-polaritonic thin films," Phys. Rev. B 75, 075422-5 (2007).

[32] R. W. Waynant, I. K. Ilev, and I. Gannot, "Mid-infrared laser applications in medicine and biology," Philos. T. R. Soc. Lond. A 359 635-644 (2001).

[33] B. Mizaikoff, "Waveguide-enhanced mid-infrared chem/bio sensors ," Chem. Soc. Rev. 42, 8683-8699 (2013).

[34] J. M. Bakker, L. M. Aleese, G. Meijer, and G. von Helden, “Fingerprint IR spectroscopy to probe amino acid conformations in the gas phase," Phys. Rev. Lett. 91, 203003 (2003).

[35] R. Assendorp, P. R. Wesselius, D. C. B. Whittet, and T. Prusti, "A study of the Chamaeleon I Dark Cloud and T-association - II: High resolution IRAS maps around HD97048 and HD97300," Mon. Not. R. Astron. Soc. 247, 624-631 (1990).

[36] H. L. Johnson, and W. W. Morgan, "Fundamental stellar photometry for standards of spectral type on the revised system of the Yerkes spectral atlas," Astrophys. J. 117, 313-352 (1953).

[37] H. U. Kaufl, "Ground-based astronomy in the 10 and $20 \mu \mathrm{m}$ atmospheric windows at ESO - scientific potential at present and in the future," The Messenger 73, 8-12 (1993).

[38] P. Yeh, Optical waves in layered media (Wiley-Interscience, Hoboken, 2005).

[39] P. Yeh, A. Yariv, and C. S. Hong, "Electromagnetic propagation in periodic stratified media. I. General theory," J. Opt. Soc. Am. 67, 423-438 (1977).

[40] A. Yariv, and P. Yeh, "Electromagnetic propagation in periodic stratified media. II. Birefringence, phase matching, and $x$-ray lasers," J. Opt. Soc. Am. 67, 438-447 (1977).

[41] S. Foteinopoulou, and C. M. Soukoulis, "Electromagnetic wave propagation in two-dimensional photonic crystals: A study of anomalous refractive effects," Phys. Rev. B 72, 165112 (2005).

[42] J. D. Joannopoulos, S. G. Johnson, J. N. Winn, and R. D. Meade, Photonic crystals: Molding the flow of light (Princeton University Press, Princeton, 2008).

[43] P. Markos, and C. M. Soukoulis, From Electrons to Photonic Crystals and Left-Handed Materials (Princeton University Press, Princeton, 2008).

[44] S. Foteinopoulou, M. Kafesaki, E. N. Economou, and C. M. Soukoulis, "Two-dimensional polaritonic photonic crystals as terahertz uniaxial metamaterials," Phys. Rev. B 84, 035128 (2011).

[45] S. Foteinopoulou, "Photonic crystals as metamaterials," Physica B 407, 4056 (2012)

[46] J. D. Jackson, Classical Electrodynamics (Third Edition, John Wiley and Sons, Hoboken, 1998).

[47] D. E. Knuth, The art of computer programming vol. 2 (Third edition, Addison-Wesley, Boston, 1998). 